PROCEEDINGS OF THE

AMERICAN MATHEMATICAL SOCIETY

Volume 138, Number 2, February 2010, Pages 395-404

S 0002-9939(09)10070-9

Article electronically published on October 2, 2009

\title{
EICHLER COHOMOLOGY THEOREM FOR AUTOMORPHIC FORMS OF SMALL WEIGHTS
}

\author{
MARVIN KNOPP AND HENOK MAWI \\ (Communicated by Wen-Ching Winnie Li)
}

\begin{abstract}
Let $\Gamma$ be an $H$-group. In 1974 Marvin Knopp conjectured that the Eichler cohomology group, with base space taken to be the set of all functions holomorphic in the upper half-plane, of polynomial growth at the real line (including $\infty$ ), and with a weight $k$,multiplier system $v$ linear fractional action of $\Gamma$, is isomorphic to the space of cusp forms on $\Gamma$ of weight $2-k$ and multiplier system $\bar{v}$, in the range $0<k<2$. In this article the authors prove the conjecture by making essential use of Hans Petersson's "principal parts condition" for automorphic forms (1955).
\end{abstract}

\section{INTRODUCTION}

Let $\mathbb{C}$ be the set of complex numbers and $\mathbb{R}$ be the set of real numbers. If

$$
S L(2, \mathbb{R})=\left\{\left(\begin{array}{ll}
a & b \\
c & d
\end{array}\right): a, b, c, d \in \mathbb{R}, a d-b c=1\right\}
$$

and $M=\left(\begin{array}{ll}a & b \\ c & d\end{array}\right)$ is in $S L(2, \mathbb{R})$, then a linear fractional transformation $\gamma$ defined by

$$
z \longmapsto \gamma z:=\frac{a z+b}{c z+d},
$$

where $z \in \mathbb{C}, a, b, c, d \in \mathbb{R}$ and $a d-b c=1$, can be identified with $\pm M$.

If $\Gamma$ is a subgroup of the group of linear fractional transformations and $\mathbb{H}=\{z \in$ $\mathbb{C}: \operatorname{Im} z>0\}$, then $\Gamma$ acts on $\mathbb{H}$ as above. $\Gamma$ is said to be a Fuchsian group of the first kind if it acts discontinuously on $\mathbb{H}$ and is discontinuous at no point of $\mathbb{R}$, and it is called an $H$ group if it is a Fuchsian group of the first kind which is finitely generated and contains translations.

Notation. For $M=\left(\begin{array}{ll}a & b \\ c & d\end{array}\right) \in \Gamma$ and $z \in \mathbb{C}$ we write $j(M, z):=c z+d$.

Let $U=\{z \in \mathbb{C}:|z|<1\}$ and let $k$ be any real number. A function $v: \Gamma \rightarrow \partial U$ for which

$$
v\left(M_{1} M_{2}\right) j\left(M_{1} M_{2}, z\right)^{k}=v\left(M_{1}\right) v\left(M_{2}\right) j\left(M_{1}, M_{2} z\right)^{k} j\left(M_{2}, z\right)^{k}
$$

Received by the editors November 18, 2008

2000 Mathematics Subject Classification. Primary 11F12, 11F75.

Key words and phrases. Automorphic forms, automorphic integrals, Eichler cohomology. 
is called a multiplier system for $\Gamma$ with weight $k$. We determine the branch of $j(M, z)^{k}$ by means of the convention

$$
w^{k}=|w|^{k} e^{i k \arg w}, \quad-\pi \leq \arg w<\pi .
$$

Let $i \infty=q_{0}, q_{1}, \ldots, q_{n}$ be the inequivalent parabolic cusps of a fundamental region $\Re$ of an Hgroup $\Gamma$ with multiplier system $v$ of weight $k$. Suppose also that for $0 \leq t \leq n$, the stabilizer group $\Gamma_{q_{t}}$ of $q_{t}$ is given by $\Gamma_{q_{t}}=\left\langle Q_{t},-I\right\rangle$. For any $M \in \Gamma$ we define the slash operator $\left.\right|_{k} ^{v}$ acting on a function $f: \mathbb{H} \rightarrow \mathbb{C}$ by

$$
\left.f\right|_{k} ^{v} M=\bar{v}(M) j(M, z)^{-k} f(M z),
$$

where the bar on $v$ is as usual complex conjugation.

For $t=0,1, \ldots, n$ put $v\left(Q_{t}\right)=e^{2 \pi i \kappa_{t}}$ with $\kappa_{t}$ normalized so that $0 \leq \kappa_{t}<1$. If $F$ is meromorphic on $\mathbb{H}$ with only finitely many poles in $\Re$ and satisfies

$$
\left.F\right|_{k} ^{v} M=F
$$

for every $M \in \Gamma$, then at each finite cusp, $q_{t}, 1 \leq t \leq n, F$ has a Fourier expansion (see [4) given by

$$
F(z)=\left(z-q_{t}\right)^{-k} \sum_{m=-\infty}^{\infty} a_{m}(t) \exp \left\{\frac{-2 \pi i\left(m+\kappa_{t}\right)}{\lambda_{t}\left(z-q_{t}\right)}\right\},
$$

valid for $\operatorname{Imz}>y_{t}$; and at the infinite cusp $q_{0}=i \infty, F$ has a similar expansion, given by

$$
F(z)=\sum_{m=-\infty}^{\infty} a_{m}(0) \exp \left\{\frac{2 \pi i\left(m+\kappa_{0}\right) z}{\lambda_{0}}\right\},
$$

valid for $\operatorname{Im} \tau>y_{0}$.

Definition 1.1. Suppose $F$ is meromorphic in $\mathbb{H}$ and satisfies the transformation law (1.2) for all $M \in \Gamma$, where $\Gamma$ is an $H$ group. We call $F$ an automorphic form of weight $k$ and multiplier system $v$ if the expansions in (1.3) and (1.4) of $F$ all contain at most finitely many terms with $m<0$. We shall denote the set of all automorphic forms by $\{\Gamma, k, v\}$.

Definition 1.2. Let $F \in\{\Gamma, k, v\}$. Suppose in addition that $F$ is holomorphic in $\mathbb{H}$ and has only terms with $m+\kappa_{t} \geq 0$ in (1.3) for $t=1,2, \ldots, n$ and $m+\kappa_{0} \geq 0$ in (1.4). Then we call $F$ an entire form. If there are only terms with $m+\kappa_{t}>0$ for $t=0,1, \ldots, n$, we call $F$ a cusp form. The set of entire forms and the set of cusp forms are denoted by $C^{+}(\Gamma, k, v)$ and $C^{0}(\Gamma, k, v)$, respectively.

Let $\mathcal{P}$ be the space of functions $g$ which are holomorphic in $\mathbb{H}$ and which satisfy the growth condition

$$
|g(z)|<K\left(|z|^{\rho}+y^{-\sigma}\right), \quad y=\operatorname{Im} z>0,
$$

for some positive constants $K, \rho$ and $\sigma$. Suppose to each $M \in \Gamma$ we associate a $g_{M} \in \mathcal{P}$. The collection $\left\{g_{M}: M \in \Gamma\right\}$ is said to be a cocycle in weight $-k$ and multiplier system $v$ if

$$
g_{M_{1} M_{2}}=\left.g_{M_{1}}\right|_{-k} ^{v} M_{2}+g_{M_{2}}, \quad \text { for all } M_{1}, M_{2} \in \Gamma .
$$

A coboundary is a cocycle $\left\{g_{M}: M \in \Gamma\right\}$ for which there exists $g \in \mathcal{P}$ such that

$$
g_{M}=\left.g\right|_{-k} ^{v} M-g, \quad \text { for all } M \in \Gamma \text {, }
$$


and a cocycle is called parabolic if for all $t, 0 \leq t \leq n$, there exists a $g_{t} \in \mathcal{P}$ such that

$$
g_{Q_{t}}=\left.g_{t}\right|_{-k} ^{v} Q_{t}-g_{t} .
$$

Definition 1.3. The Eichler cohomology group $H_{k, v}^{1}(\Gamma, \mathcal{P})$ is defined to be the vector space of cocycles of weight $-k$ modulo coboundaries. We also define $\widetilde{H}_{k, v}^{1}(\Gamma, \mathcal{P})$ to be the subgroup of $H_{k, v}^{1}(\Gamma, \mathcal{P})$ which is formed by taking the space of parabolic cocycles modulo coboundaries.

Definition 1.4. If $F$ is a function meromorphic in $\mathbb{H}$ such that

$$
\left.F\right|_{-k} ^{v} M-F \in \mathcal{P}, \quad \text { for all } M \in \Gamma
$$

and for each $t, 1 \leq t \leq n$, there exists an integer $m_{t}$ such that

$$
\exp \left\{2 \pi i\left(m_{t}+\kappa_{t}\right) / \lambda_{j}\left(z-q_{t}\right)\right\} F(z)
$$

has a limit as $z \rightarrow q_{t}$ within $\Re$ and there is also an integer $m_{0}$ such that

$$
\exp \left\{2 \pi i\left(m_{0}+\kappa_{0}\right) / \lambda_{0}\right\} F(z)
$$

has a limit as $z \rightarrow i \infty$ within $\Re$, then we call $F$ an automorphic integral of weight $-k$.

Notice that an automorphic form is an automorphic integral and in fact if $F$ is an automorphic integral (see [5]) and for each $M \in \Gamma$ we set $g_{M}=\left.F\right|_{-k} ^{v} M-F$, then we can show that the collection $\left\{g_{M}: M \in \Gamma\right\}$ is actually a cocycle. It is therefore plausible and in fact possible to consider automorphic integrals and to determine the structure of both $H_{k, v}^{1}(\Gamma, \mathcal{P})$ and $\widetilde{H}_{k, v}^{1}(\Gamma, \mathcal{P})$. In particular if, in place of $\mathcal{P}$, we take $P_{k}(k \in \mathbb{Z}, k \geq 0)$, the set of polynomials of degree less than or equal to $k$, and we let $\Gamma$ be an $H$ group, then the structure of $H_{k, v}^{1}\left(\Gamma, P_{k}\right)$ and $\widetilde{H}_{k, v}^{1}\left(\Gamma, P_{k}\right)$ can be completely determined (see 1], 2, 3, 8). Also ([6, Theorem 1) states that if $k \geq 0$ or $k \leq-2$, then

$$
C^{0}(\Gamma, k+2, v) \cong \widetilde{H}_{k, v}^{1}(\Gamma, \mathcal{P}) .
$$

In [6] it is also shown that for any real number $k$

$$
H_{k, v}^{1}(\Gamma, \mathcal{P})=\widetilde{H}_{k, v}^{1}(\Gamma, \mathcal{P}) .
$$

Combining this with (1.5) we find that

$$
C^{0}(\Gamma, k+2, v) \cong H_{k, v}^{1}(\Gamma, \mathcal{P}),
$$

for $k \geq 0$ and $k \leq-2, k \in \mathbb{R}$. In the same paper Knopp conjectured that (1.5) holds true even when $-2<k<0$, and in 12, Wang proved this conjecture for the interval $-2<k<-1$, but only under the additional restriction that $C^{0}(\Gamma, k+2, \bar{v})=0$. The latter restriction is essential to Wang's proof, as it suffices (and it is necessary, as well, as it turns out) for the construction he requires of automorphic forms of weight $k,-2<k<-1$, with preassigned principal parts at the parabolic cusps of $\Gamma$. The construction follows from Selberg's well known work on the nonanalytic Poincaré series (i.e. with Hecke convergence factor), the Fourier coefficients of which involve Selberg's Kloosterman zeta function [11.

The same restriction is actually present in the proof of (1.5) given in [6], appearing as the exclusion of the interval $-2<k<0$. When $k \leq-2$, the space $C^{0}(\Gamma, k+2, \bar{v})=\{0\}$, and this fact is used in [6], together with a Mittag-Leffler result for automorphic forms of weight $k \geq 2$ on $\Gamma$. This Mittag-Leffler result (the 
existence of an automorphic form of fixed weight $k \geq 2$ and fixed multiplier system with preassigned principal parts at a finite number of arbitrarily chosen points in a fundamental region) is a direct consequence of the special case of Petersson's principal parts conditions [10] obtained when $C^{0}(\Gamma, k+2, \bar{v})=\{0\}$. For $k \geq 0$, the proof of (1.5) in 6] relies upon work of Niebur relating $C^{0}(\Gamma, k+2, \bar{v})$ to parabolic cocycles in $\widetilde{H}_{k, v}^{1}(\Gamma, \mathcal{P})[9$; in fact the construction in [6] of the isomorphism between these two spaces in the case $k \geq 0$ is not possible without the results of [9].

In this paper we prove Knopp's conjecture fully. As the referee has pointed out the proof of (1.5) given here is valid for all real $k$, not simply for the previously excluded interval $-2<k<0$. The validity of this proof for all real $k$ depends upon our application of the full strength of the Petersson principal parts condition, that is, including an application to those cases in which $C^{0}(\Gamma, k+2, \bar{v}) \neq\{0\}$. However, as the results of [9] are not available for $k<0$, the canonical isomorphism of (1.5) is different here from that given in [6] for the case $k \geq 0$. Thus when $k \geq 0$ there are (at least) two different versions of the isomorphism.

We thank the referee for a careful reading of our manuscript and for the insightful suggestions which led to significant improvements in our exposition, especially the proof of Proposition 3.2 .

\section{Statement of the MAin Result}

Theorem 2.1 (Main result). If $k \in \mathbb{R}$ and $v$ is a multiplier system of weight $-k$, then $C^{0}(\Gamma, k+2, \bar{v})$ is isomorphic to $\widetilde{H}_{k, v}^{1}(\Gamma, \mathcal{P})$.

To prove this theorem let us first prove the following:

Lemma 2.2. Let $G \in C^{0}(\Gamma, k+2, \bar{v})$ and $z \in \mathbb{H}$. Put

$$
g(z)=\left[\int_{i \infty}^{z} G(\tau)(\tau-\bar{z})^{k} d \tau\right]^{-}
$$

Then

$$
\left.g\right|_{-k} ^{v} M-g=\left[\int_{M^{-1}(i \infty)}^{i \infty} G(\tau)(\tau-\bar{z})^{k} d \tau\right]^{-},
$$

for all $M \in \Gamma$. Moreover if for each $M \in \Gamma$ we set

$$
g_{M}(z)=\left[\int_{M^{-1}(i \infty)}^{i \infty} G(\tau)(\tau-\bar{z})^{k} d \tau\right]^{-},
$$

then the collection $\left\{g_{M}: M \in \Gamma\right\}$ is a cocycle in $\mathcal{P}$.

Proof. First we have

$$
\begin{aligned}
\left.g\right|_{-k} ^{v} M-g & =\bar{v}(M) j(M, z)^{k} g(M z)-g(z) \\
& =\left[v(M) j(M, \bar{z})^{k} \int_{i \infty}^{M z} G(\tau)(\tau-M \bar{z})^{k} d \tau-\int_{i \infty}^{z} G(\tau)(\tau-\bar{z})^{k} d \tau\right]^{-} .
\end{aligned}
$$


Then using the substitution $\tau \rightarrow M \tau$ in the first integral and the fact that $G \in$ $C^{0}(\Gamma, k+2, \bar{v})$, we obtain

$$
\begin{gathered}
\left.g\right|_{-k} ^{v} M-g=\left[\int_{M^{-1}(i \infty)}^{z} j(M, \bar{z})^{k} j(M, \tau)^{k} G(\tau)(M \tau-M \bar{z})^{k} d \tau-\int_{i \infty}^{z} G(\tau)(\tau-\bar{z})^{k} d \tau\right]^{-} \\
=\left[\int_{M^{-1}(i \infty)}^{z} j(M, \bar{z})^{k} j(M, \tau)^{k} G(\tau)\left(\frac{\tau-\bar{z}}{j(M, \tau) j(M, \bar{z})}\right)^{k} d \tau-\int_{i \infty}^{z} G(\tau)(\tau-\bar{z})^{k} d \tau\right]^{-} .
\end{gathered}
$$

By comparing arguments and observing that

$$
\frac{\tau-\bar{z}}{j(M, \tau) j(M, \bar{z})}=M \tau-M \bar{z}
$$

is in $\mathbb{H}$, we can show that

$$
\left(\frac{\tau-\bar{z}}{j(M, \tau) j(M, \bar{z})}\right)^{k}=\frac{(\tau-\bar{z})^{k}}{j(M, \tau)^{k} j(M, \bar{z})^{k}}
$$

and hence conclude by Cauchy's theorem that

$$
\left.g\right|_{-k} ^{v} M-g=\left[\int_{M^{-1} i \infty}^{i \infty} G(\tau)(\tau-\bar{z})^{k} d \tau\right]^{-} .
$$

From this it follows that $\left\{g_{M}: M \in \Gamma\right\}$ is a cocycle.

We now define a mapping

$$
\eta: C^{0}(\Gamma, k+2, \bar{v}) \longrightarrow H_{k, v}^{1}(\Gamma, \mathcal{P})
$$

by $\eta(G)=\left\langle g_{M}: M \in \Gamma\right\rangle$, where $\left\langle g_{M}: M \in \Gamma\right\rangle$ is the class determined by the cocycle $\left\{g_{M}: M \in \Gamma\right\}$ constructed in the previous lemma. Clearly $\eta$ is a linear mapping. To prove our main result it suffices to show that $\eta$ is an isomorphism onto $H_{k, v}^{1}(\Gamma, \mathcal{P})$.

To prove that $\eta$ is one-to-one, assume that $G \in \operatorname{ker} \eta$. This means $\eta(G)=\left\langle g_{M}\right.$ : $M \in \Gamma\rangle$ is a coboundary. Thus there exists $h \in \mathcal{P}$ such that

$$
g_{M}=\left.h\right|_{-k} ^{v} M-h
$$

for all $M \in \Gamma$. But then with $g$ defined as in (2.1), we obtain

$$
\left.(g-h)\right|_{-k} ^{v} M-(g-h)=0 \quad \text { for all } M \in \Gamma \text {. }
$$

As usual let

$$
\partial_{\bar{z}}:=\frac{1}{2}\left(\partial_{x}+i \partial_{y}\right)
$$

Then, since $h$ and $G$ are holomorphic,

$$
\partial_{\bar{z}}(g(z) G(z))=|G(z)|^{2}(\bar{z}-z)^{k}
$$

and

$$
\partial_{\bar{z}}(h(z) G(z))=0 .
$$

Thus

$$
\iint_{\Re}|G(z)|^{2}(\bar{z}-z)^{k} d x d y=\iint_{\Re} \partial_{\bar{z}} G(g-h) d x d y .
$$

Then Stokes's theorem applied to the right hand side of (2.3) gives

$$
\iint_{\Re}|G(z)|^{2}(\bar{z}-z)^{k} d x d y=\frac{-i}{2} \int_{\partial \Re} G(g-h) d z .
$$


Using the transformation property of $G(g-h)$ we can show that the right hand side of (2.4) is zero, from which we conclude that $G \equiv 0$. For details of the proof we refer the reader to [7. This proves that $\operatorname{Ker} \eta=0$ and thus that $\eta$ is one-to-one.

To complete the proof of the main result it thus remains to prove that $\eta$ is onto. To do so we need some results from the next section.

\section{Petersson's PRincipal parts CONDition AND RELAted RESUlts}

Let $k \in \mathbb{R}$. Let $\left\{\varphi_{1}, \ldots, \varphi_{d}\right\}$ be a basis of $C^{0}(\Gamma, k+2, \bar{v})$ whose expansions at the finite cusps $q_{t}$ are given by

$$
\varphi_{l}(z)=\left(z-q_{t}\right)^{-k-2} \sum_{m+\kappa_{t}>0}^{m=\infty} a_{m}^{(l)}(t) \exp \left\{\frac{-2 \pi i\left(m+\kappa_{t}\right)}{\lambda_{t}\left(z-q_{t}\right)}\right\}, \text { for } l=1, \ldots, d,
$$

and whose expansions at $q_{0}$ are given by

$$
\varphi_{l}(z)=\sum_{m+\kappa_{o}>0}^{m=\infty} a_{m}^{(l)}(0) \exp \left\{\frac{2 \pi i\left(m+\kappa_{0}\right) z}{\lambda_{0}}\right\} .
$$

Let $\kappa_{t}^{+} \equiv \kappa_{t} \bmod 1,0<\kappa_{t}^{+} \leq 1, \kappa_{t}^{\prime}=1-\kappa_{t}^{+}$, and let $\mu_{t} \geq 0, t=0,1, \ldots, n$.

Proposition 3.1 (Petersson, 1955). With the above setting, there exists $g \in$ $\{\Gamma,-k, v\}$, holomorphic in $\mathbb{H}$ and whose expansions at the cusps are given by

$$
g(z)=\left(z-q_{t}\right)^{k} \sum_{m=-\mu_{t}}^{m=\infty} b_{m}(t) \exp \left\{\frac{-2 \pi i\left(m+\kappa_{t}^{+}\right)}{\lambda_{t}\left(z-q_{t}\right)}\right\}, \quad \text { for } 1 \leq t \leq n,
$$

and by

$$
g(z)=\sum_{m=-\mu_{0}}^{m=\infty} b_{m}(0) \exp \left\{\frac{2 \pi i\left(m+\kappa_{0}^{+}\right) z}{\lambda_{0}}\right\}, \quad \text { for } t=0,
$$

if and only if

$$
\sum_{t=0}^{n} \sum_{m=1}^{\mu_{t}} b_{-m}(t) a_{m-1}^{(l)}(t)=0
$$

for each $l=1, \ldots, d$.

For the proof of the above proposition see [10] (pp. 388-389).

Proposition 3.2. If $\left\{\varphi_{1}, \ldots, \varphi_{d}\right\}$ is as above and the expansion of $\varphi_{l}$ at the infinite cusp is given by (3.1), then there exist natural numbers $w_{1}<\ldots<w_{d}$ such that the $d \times d$ matrix $C=\left[c_{l j}\right]$, where $c_{l j}=a_{w_{j}}^{(l)}:=a_{w_{j}}^{(l)}(0)$, is nonsingular.

Proof. Consider the element in the dual $C^{0}(\Gamma, k+2, \bar{v})^{*}$ of $C^{0}(\Gamma, k+2, \bar{v})$ given by $a_{w}(\phi)=w^{t h}$ coefficient of $\phi$ in the expansion of $\phi$ at $i \infty$.

Then given an inner product $\langle$,$\rangle on C^{0}(\Gamma, k+2, \bar{v})$ there exist $\phi_{w} \in C^{0}(\Gamma, k+2, \bar{v})$ such that

$$
a_{w}(\phi)=\left\langle\phi_{w}, \phi\right\rangle \quad \text { for all } \phi .
$$

Clearly $\left\langle\phi_{w}, \phi\right\rangle=0$ for all $w$ iff $\phi \equiv 0$. Thus $\left\{\phi_{w}\right\}$ spans $C^{0}(\Gamma, k+2, \bar{v})$ with

$$
\phi=\sum_{w=1}^{\infty}\left\langle\phi_{w}, \phi\right\rangle \phi_{w} .
$$


Now if $a \in C^{0}(\Gamma, k+2, \bar{v})^{*}$ is any linear functional, then

$$
a(\phi)=\sum_{w=1}^{\infty} a\left(\phi_{w}\right)\left\langle\phi_{w}, \phi\right\rangle=\sum_{w=1}^{\infty} a\left(\phi_{w}\right) a_{w}(\phi),
$$

and therefore $\left\{a_{w}\right\}$ generate $C^{0}(\Gamma, k+2, \bar{v})^{*}$. Choose a basis $\left\{a_{w_{1}}, \ldots, a_{w_{d}}\right\}, w_{1}<$ $\ldots<w_{d}$ and construct the matrix $C=\left[c_{l j}\right]$ with $c_{l j}=a_{w_{j}}\left(\varphi_{l}\right)=a_{w_{j}}^{l}(0)=: a_{w_{j}}^{l}$. Then $C$ is nonsingular. Indeed, otherwise there exist $\alpha_{1}, \ldots, \alpha_{d}$ not all zero such that

$$
\alpha_{1} C_{1}+\ldots+\alpha_{d} C_{d}=0,
$$

where $C_{l}=\left(c_{l 1}, \ldots, c_{l d}\right)$ for $l=1, \ldots, d$. If without loss of generality we assume that $\alpha_{1} \neq 0$, then this would mean $a_{w_{1}}$ is a linear combination of $a_{w_{2}}, \ldots, a_{w_{d}}$, and this is a contradiction.

Proposition 3.3. Let $k \in \mathbb{R}$. Given poles of prescribed principal parts $P_{t}$ at the cusp $q_{t}$ for $t=0,1, \ldots, n$, there exists $g \in\{\Gamma,-k, v\}$ whose principal part at the finite cusp $q_{t}, 1 \leq t \leq n$, is $P_{t}$ and whose principal part at the infinite cusp $q_{0}$ differs from $P_{0}$ by at most $d$ terms where $d=\operatorname{dim} C^{0}(\Gamma, k+2, \bar{v})$.

Proof. Suppose $\left\{\varphi_{1}, \ldots, \varphi_{d}\right\}$ is a basis of $C^{0}(\Gamma, k+2, \bar{v})$. Suppose

$$
\varphi_{l}(z)=\sum_{m+\kappa_{0}>0} a_{m}^{(l)} \exp \left\{\frac{2 \pi i\left(m+\kappa_{0}\right) z}{\lambda_{0}}\right\}
$$

is the expansion at $q_{0}$ of $\varphi_{l}$. Let $w_{1}<\ldots<w_{d}$ and $C$ be as in Proposition 3.2. We shall construct $g \in\{\Gamma,-k, v\}$ whose principal part at $q_{t}$ for $1 \leq t \leq n$ is $P_{t}$ and whose principal part at $q_{0}$ is given by

$$
g(z)=P_{0}+\sum_{j=1}^{d} a_{w_{j}} \exp \left\{\frac{-2 \pi i\left(w_{j}+\kappa_{0}^{\prime}\right) z}{\lambda_{0}}\right\}
$$

where $a_{w_{j}}$ are to be determined.

By using Proposition 3.1 we see that the necessary and sufficient condition for $g$ to exist is

$$
C A_{w}=B
$$

where $A_{w}=\left(a_{w_{1}}, \ldots, a_{w_{d}}\right)$ and $B=\left(b_{1}, \ldots, b_{d}\right)$ with $b_{j}=-\sum_{t=0}^{n}\left(\operatorname{Res}\left(P_{t} \varphi_{j}\right), q_{t}\right)$. But since $C$ is nonsingular, (3.5) has a unique solution for $A_{w}=\left(a_{w_{1}}, \ldots, a_{w_{d}}\right)$. With these values for $a_{w_{1}}, \ldots, a_{w_{d}}$ Petersson's principal parts condition is satisfied by $g$, and hence $g$ exists.

\section{Proof of the main Result}

To complete the proof of the main result it remains to establish that the linear map $\eta$ defined by (2.2) is onto.

To this end we will need the following proposition. For the proof one can refer to [6], Theorem 3, pp. 613-614 and pp. 619-620.

Proposition 4.1. Let $k$ be any real number and $v$ be a multiplier system of weight $-k$. Suppose $\left\{g_{M}: M \in \Gamma\right\}$ is a parabolic cocycle of weight $-k$ in $\mathcal{P}$; that is,

$$
g_{M_{1} M_{2}}=\left.g_{M_{1}}\right|_{-k} ^{v} M_{2}+g_{M_{2}} \text {, for all } M_{1}, M_{2} \in \Gamma,
$$


and for all $t, 0 \leq t \leq n$, there exists a $g_{t}$ in $\mathcal{P}$ such that

$$
g_{Q_{t}}=\left.g_{t}\right|_{-k} ^{v} Q_{t}-g_{t} .
$$

Then there exists a function $\Phi$, holomorphic in $\mathbb{H}$, such that

$$
\left.\Phi\right|_{-k} ^{v} M-\Phi=g_{M} \text { for all } M \in \Gamma,
$$

and which has expansions at the parabolic cusps $q_{t}, 0 \leq t \leq n$, of the form

$$
\begin{aligned}
& \Phi(z)=g_{t}(z)+\left(z-q_{t}\right)^{k} \sum_{m=-m_{t}}^{m=\infty} a_{m}(t) \exp \left\{\frac{-2 \pi i\left(m+\kappa_{t}\right)}{\lambda_{t}\left(z-q_{t}\right)}\right\}, \text { for } 1 \leq t \leq n, \\
& \Phi(z)=g_{0}(z)+\sum_{m=-m_{0}}^{m=\infty} a_{m}(0) \exp \left\{\frac{2 \pi i\left(m+\kappa_{0}\right) z}{\lambda_{0}}\right\}, \text { for } t=0 .
\end{aligned}
$$

Proof of Theorem 2.1. Assume that $\operatorname{dim} C^{0}(\Gamma, k+2, \bar{v})=d$. Since $\eta$ is one-to-one and $H_{k, v}^{1}(\Gamma, \mathcal{P}) \cong \widetilde{H}_{k, v}^{1}(\Gamma, \mathcal{P})$, it suffices to show that $\operatorname{dim} \widetilde{H}_{k, v}^{1}(\Gamma, \mathcal{P})$ is at most $d$.

If $\operatorname{dim} C^{0}(\Gamma, k+2, \bar{v})=0$, then (3.2) imposes no condition, and so there exists a $g \in\{\Gamma,-k, v\}$ with any preassigned principal parts at each of the cusps. Thus given a parabolic cocycle $\left\{g_{M}: M \in \Gamma\right\}$, we obtain $\Phi$ whose expansion at the cusps has principal parts as given in Proposition 4.1. We then use Proposition 3.2 to obtain a $g \in\{\Gamma, k, v\}$ whose expansions at the cusps have principal parts which agree with those of $\Phi$. Now set $\Phi^{*}=\Phi-g$. Then $\left.\Phi^{*}\right|_{-k} ^{v} M-\Phi^{*}=g_{M}$ for all $M \in \Gamma$ and $\Phi^{*} \in \mathcal{P}$. (See [5].) Thus every parabolic cocycle is a coboundary, and hence the dimension of $\widetilde{H}_{k, v}^{1}(\Gamma, \mathcal{P})$ is zero. Therefore

$$
C^{0}(\Gamma, k+2, \bar{v}) \cong \widetilde{H}_{k, v}^{1}(\Gamma, \mathcal{P})=\{0\},
$$

and this completes the proof of Theorem 2.1 when $d=0$.

Now suppose $\operatorname{dim} C^{0}(\Gamma, k+2, \bar{v})=d>0$. Since $\eta$ is one-to-one and $H_{k, v}^{1}(\Gamma, \mathcal{P}) \cong$ $\widetilde{H}_{k, v}^{1}(\Gamma, \mathcal{P})$, we know that there exist at least $d$ linearly independent elements in $\widetilde{H}_{k, v}^{1}(\Gamma, \mathcal{P})$. Let $d$ of these linearly independent elements be

$$
\left\langle g_{M}^{1}: M \in \Gamma\right\rangle, \ldots,\left\langle g_{M}^{d}: M \in \Gamma\right\rangle,
$$

and let

$$
\left\{g_{M}^{1}: M \in \Gamma\right\}, \ldots,\left\{g_{M}^{d}: M \in \Gamma\right\}
$$

be respective representatives. Corresponding to each of these parabolic cocycles, by using Proposition 4.1, we obtain a $\Phi_{i}, 1 \leq i \leq d$, holomorphic in $\mathbb{H}$ such that

$$
\begin{aligned}
& \Phi_{i}(z)=g_{t}^{i}(z)+\left(z-q_{t}\right)^{k} \sum_{m=-m_{t}^{i}}^{m=\infty} a_{m}^{i}(t) \exp \left\{\frac{-2 \pi i\left(m+\kappa_{t}\right)}{\lambda_{t}\left(z-q_{t}\right)}\right\}, \text { for } 1 \leq t \leq n, \\
& \Phi_{i}(z)=g_{0}^{i}(z)+\sum_{m=-m_{0}^{i}}^{m=\infty} a_{m}^{i}(0) \exp \left\{\frac{2 \pi i\left(m+\kappa_{0}\right) z}{\lambda_{0}}\right\} .
\end{aligned}
$$

By Proposition 3.3 for each $\Phi_{i}$ there exists $f_{i}, f_{i} \in\{\Gamma,-k, v\}$, such that except possibly for $d$ terms in the expansion of $f_{i}$ at the infinite cusp, $f_{i}$ has exactly the 
same principal parts as $\Phi_{i}$ in all the expansions at the cusps. Thus after renaming coefficients,

$$
\begin{aligned}
& \Phi_{i}^{*}(z)=g_{t}^{i}(z)+\left(z-q_{t}\right)^{k} \sum_{m=0}^{m=\infty} a_{m}^{i}(t) \exp \left\{\frac{-2 \pi i\left(m+\kappa_{t}\right)}{\lambda_{t}\left(z-q_{t}\right)}\right\}, \quad \text { for } 1 \leq t \leq n, \\
& \Phi_{i}^{*}(z)=g_{0}^{i}(z)+\sum_{l=1}^{d} a_{-l}^{i}(0) \exp \left\{\frac{-2 \pi i\left(l+\kappa_{0}\right) z}{\lambda_{0}}\right\}+\sum_{m=0}^{m=\infty} a_{m}^{i}(0) \exp \left\{\frac{2 \pi i\left(m+\kappa_{0}\right) z}{\lambda_{0}}\right\},
\end{aligned}
$$

where $\Phi_{i}^{*}=\Phi_{i}-f_{i}$.

The $\Phi_{i}^{*}$ 's are linearly independent. Indeed, otherwise there exist $\beta_{1}, \ldots, \beta_{d}$ not all zero such that

$$
\Theta=\sum_{i=1}^{d} \beta_{i} \Phi_{i}^{*}=0 .
$$

This gives $\left.\Theta\right|_{-k} ^{v} M-\Theta=0$, which in turn gives that $\sum_{i=1}^{d} \beta_{i}\left\langle g_{M}^{i}\right\rangle=0$, where we have written $\left\langle g_{M}^{i}\right\rangle$ in place of $\left\langle g_{M}^{i}: M \in \Gamma\right\rangle$ for simplicity. But this contradicts the assumption that $\left\langle g_{M}^{1}\right\rangle, \ldots,\left\langle g_{M}^{d}\right\rangle$ are linearly independent. If $\left\langle g_{M}\right\rangle \neq 0$ is now any other element in $\widetilde{H}_{k, v}^{1}(\Gamma, \mathcal{P})$, we shall show that $\left\langle g_{M}\right\rangle$ is a linear combination of $\left\langle g_{M}^{1}\right\rangle, \ldots,\left\langle g_{M}^{d}\right\rangle$. To this end, as we did above, we first obtain

$$
\begin{aligned}
& \Phi(z)=g_{t}(z)+\left(z-q_{t}\right)^{k} \sum_{m=-m_{t}}^{m=\infty} a_{m}(t) \exp \left\{\frac{-2 \pi i\left(m+\kappa_{t}\right)}{\lambda_{t}\left(z-q_{t}\right)}\right\}, \text { for } 1 \leq t \leq n, \\
& \Phi(z)=g_{0}(z)+\sum_{m=-m_{0}}^{m=\infty} a_{m}(0) \exp \left\{\frac{2 \pi i\left(m+\kappa_{0}\right) z}{\lambda_{0}}\right\}, \text { for } t=0,
\end{aligned}
$$

corresponding to $\left\langle g_{M}\right\rangle$. Once again using Proposition 3.3, we obtain $f \in\{\Gamma,-k, v\}$ such that

$$
\begin{aligned}
& \Phi^{*}(z)=g_{t}(z)+\left(z-q_{t}\right)^{k} \sum_{m=0}^{m=\infty} a_{m}(t) \exp \left\{\frac{-2 \pi i\left(m+\kappa_{t}\right)}{\lambda_{t}\left(z-q_{t}\right)}\right\}, \quad \text { for } 1 \leq t \leq n, \\
& \Phi^{*}(z)=g_{0}(z)+\sum_{l=1}^{d} a_{-l}(0) \exp \left\{\frac{-2 \pi i\left(l+\kappa_{0}\right) z}{\lambda_{0}}\right\}+\sum_{m=0}^{m=\infty} a_{m}(0) \exp \left\{\frac{2 \pi i\left(m+\kappa_{0}\right) z}{\lambda_{0}}\right\},
\end{aligned}
$$

where $\Phi^{*}=\Phi-f$.

Since the $\Phi_{i}^{*}$ 's are linearly independent we notice that the determinant of the matrix

$$
\left[\begin{array}{ccc}
a_{-1}^{1}(0) & \ldots & a_{-d}^{1}(0) \\
\vdots & \vdots & \vdots \\
a_{-1}^{d}(0) & \ldots & a_{-d}^{d}(0)
\end{array}\right]
$$

will be nonzero. Otherwise there exist $\beta_{1}, \ldots, \beta_{d}$ not all zero such that $\sum_{i=1}^{d} \beta_{i} \Phi_{i}^{*}$ has no poles at $q_{0}=i \infty$. Then $\sum_{i=1}^{d} \beta_{i} \Phi_{i}^{*} \in \mathcal{P}$. From this we can deduce that $\sum_{i=1}^{d} \beta_{i}\left\langle g_{M}^{i}\right\rangle$ is a coboundary, which is a contradiction. Thus there exist $\alpha_{1}, \ldots, \alpha_{d}$ such that

$$
a_{-p}(0)+\sum_{i=1}^{d} \alpha_{i} a_{-p}^{i}(0)=0 \quad \text { for all } p, 1 \leq p \leq d .
$$


Now define

$$
\Psi=\Phi^{*}+\sum_{i=1}^{d} \alpha_{i} \Phi_{i}^{*}
$$

Then $\Psi \in \mathcal{P}$ and has expansions at the cusps given by

$$
\begin{aligned}
& \Psi(z)=g_{t}(z)+\sum_{i=1}^{d} \alpha_{i} g_{t}^{i}(z)+\left(z-q_{t}\right)^{k} \sum_{m=0}^{m=\infty} b_{m}(t) \exp \left\{\frac{-2 \pi i\left(m+\kappa_{t}\right)}{\lambda_{t}\left(z-q_{t}\right)}\right\}, \\
& \text { for } 1 \leq t \leq n \text {, } \\
& \Psi(z)=g_{0}(z)+\sum_{i=1}^{d} \alpha_{i} g_{0}^{i}(z)+\sum_{m=0}^{m=\infty} b_{m}(0) \exp \left\{\frac{2 \pi i\left(m+\kappa_{0}\right) z}{\lambda_{0}}\right\} .
\end{aligned}
$$

Moreover,

$$
\left.\Psi\right|_{-k} ^{v} M-\Psi=g_{M}+\sum_{i=1}^{d} \alpha_{i} g_{M}^{i}
$$

Thus

$$
\left\{g_{M}+\sum_{i=1}^{d} \alpha_{i} g_{M}^{i}: M \in \Gamma\right\}
$$

is a coboundary. This proves that $\left\langle g_{M}: M \in \Gamma\right\rangle$ is indeed a linear combination of $\left\langle g_{M}^{1}: M \in \Gamma\right\rangle, \ldots,\left\langle g_{M}^{d}: M \in \Gamma\right\rangle$, and the proof is complete.

\section{REFERENCES}

[1] M. Eichler, Eine Verallgemeinerung der Abelschen Integrale, Math. Z. 67, 1957. MR0089928 $(19: 740 \mathrm{a})$

[2] R. C. Gunning, The Eichler cohomology groups and automorphic forms, Trans. Amer. Math. Soc. 100, 1961. MR0140126 (25:3549)

[3] S. Y. Husseini and M. I. Knopp, Eichler cohomology and automorphic forms, Illinois J. Math. 15, 1971. MR0285733 (44:2950)

[4] M. I. Knopp, Modular Functions in Analytic Number Theory, 2nd ed., Amer. Math. Soc. Chelsea series, 1993. MR0265287 (42:198)

[5] M. I. Knopp, On the growth of entire automorphic integrals, Results in Mathematics 8, 1985. MR828936 (87e:11065)

[6] M. I. Knopp, Some new results on the Eichler cohomology of automorphic forms, Bulletin of Amer. Math. Soc. 80, 1974. MR0344454 (49:9193)

[7] M. I. Knopp, J. Lehner and W. Raji, Eichler cohomology for generalized modular forms, International Journal of Number Theory 5, no. 6, 2009, to appear.

[8] J. Lehner, Automorphic integrals with preassigned period polynomials and the Eichler cohomology, Computers in Number Theory, Proc. Science Research Council Atlas Sympos. no. 2, Academic Press, London and New York, 1971. MR.0316387 (47:4934)

[9] D. Niebur, Construction of automorphic forms and integrals, Trans. Amer. Math. Soc. 191, 1974. MR0344196 (49:8936)

[10] Hans Petersson, Über automorphe Formen mit Singularitäten im Diskontinuitätsgebiet, Math. Annalen 129, 1955. MR0071459(17:129c)

[11] A. Selberg, On the estimation of Fourier coefficients of modular forms, Theory of Numbers, Proc. Symposium Pure Math, vol. 8, Amer. Math. Soc., Providence, RI, 1965. MR0182610 $(32: 93)$

[12] X. Wang, A conjecture on the Eichler cohomology of automorphic forms, Science in China (series A) 43, no. 7, 2000. MR1790176 (2001i:11049)

Department of Mathematics, Temple University, Philadelphia, Pennsylvania 19122

Department of Mathematics, Temple University, Philadelphia, Pennsylvania 19122 